\title{
Caution in Correcting Hyponatremia in Patients on Vasopressin
}

Rami Jambeih, M.D.

\section{Introduction}

Vasopressin is commonly used as a pressor agent in the treatment of septic shock.

- Despite the frequent use of vasopressin, few cases of hyponatremia have been reported in association with its use.

\section{Case Presentation}

* 24-year-old male presented with status epilepticus requiring mechanical ventilation.

The hospital stay was complicated by ventilator associated pneumonia and sepsis requiring vasopressin infusion to maintain his blood pressure.

- The patient developed hyposmolar hyponatremia (serum Na=126 mEq/L; serum osmolarity $=265$ Osm/L).

- Water restriction and discontinuation of vasopressin resulted in polyuria and an abrupt rise in serum sodium to $140 \mathrm{mEq} / \mathrm{L}$, mimicking diabetes insipidus.

\section{Discussion}

* Vasopressin is synthesized in the hypothalamus and secreted by the posterior pituitary gland.

* It acts on the V1 receptors on the blood vessels to induce vasoconstriction.

- It also acts on the distal tubules and collecting ducts in the kidneys via $\mathrm{V} 2$ receptors to promote water reabsorption.
Mohamad Sandid, M.D.

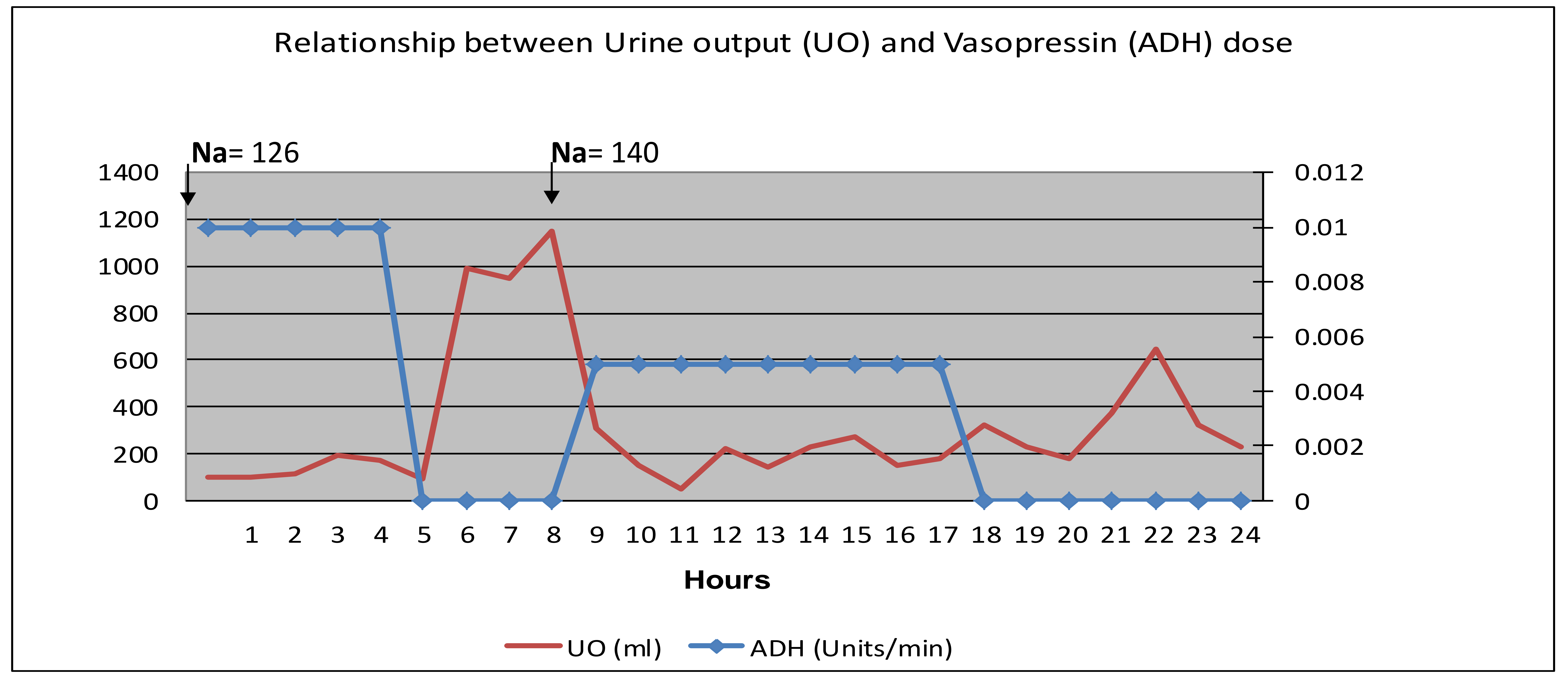

Vasopressin is used in the management of septic shock at a maximum dose of 0.04 Units/min.

* The infusion of vasopressin at this dose or higher is associated with systemic side effects, mainly cardiac ischemia and arrhythmia.

* Vasopressin also may cause hyponatremia by inducing water reabsorption and hemodilution.

In a retrospective review of 102 patients with septic shock on vasopressin, only one case of hyponatremia was reported.

\section{Conclusions}

* Although vasopressin frequently is used in the ICU, it is not frequently associated with electrolyte abnormalities.

This case reminds us that 1) vasopressin is rarely associated with hyponatremia, and 2) abrupt discontinuation of vasopressin can result in a a rapid and unexpected correction of the serum sodium.

\section{References}

- Role of vasopressin in the management of septic shock. Intensive Care Med 2004; 30(7):1276-1291.

- Effects of continuous vasopressin infusion in patients with septic shock. Ann Pharmacother 2004; 38(7-8):1117-1122.

- Multicenter randomized controlled trial of terlipressin versus sclerotherapy in the

treatment of acute variceal bleeding: The TEST study. Hepatology 2000; 32:471-476.

- Transient diabetes insipidus after discontinuation of therapeutic vasopressin. Pharmacotherapy 2004; 24(4):541-545.

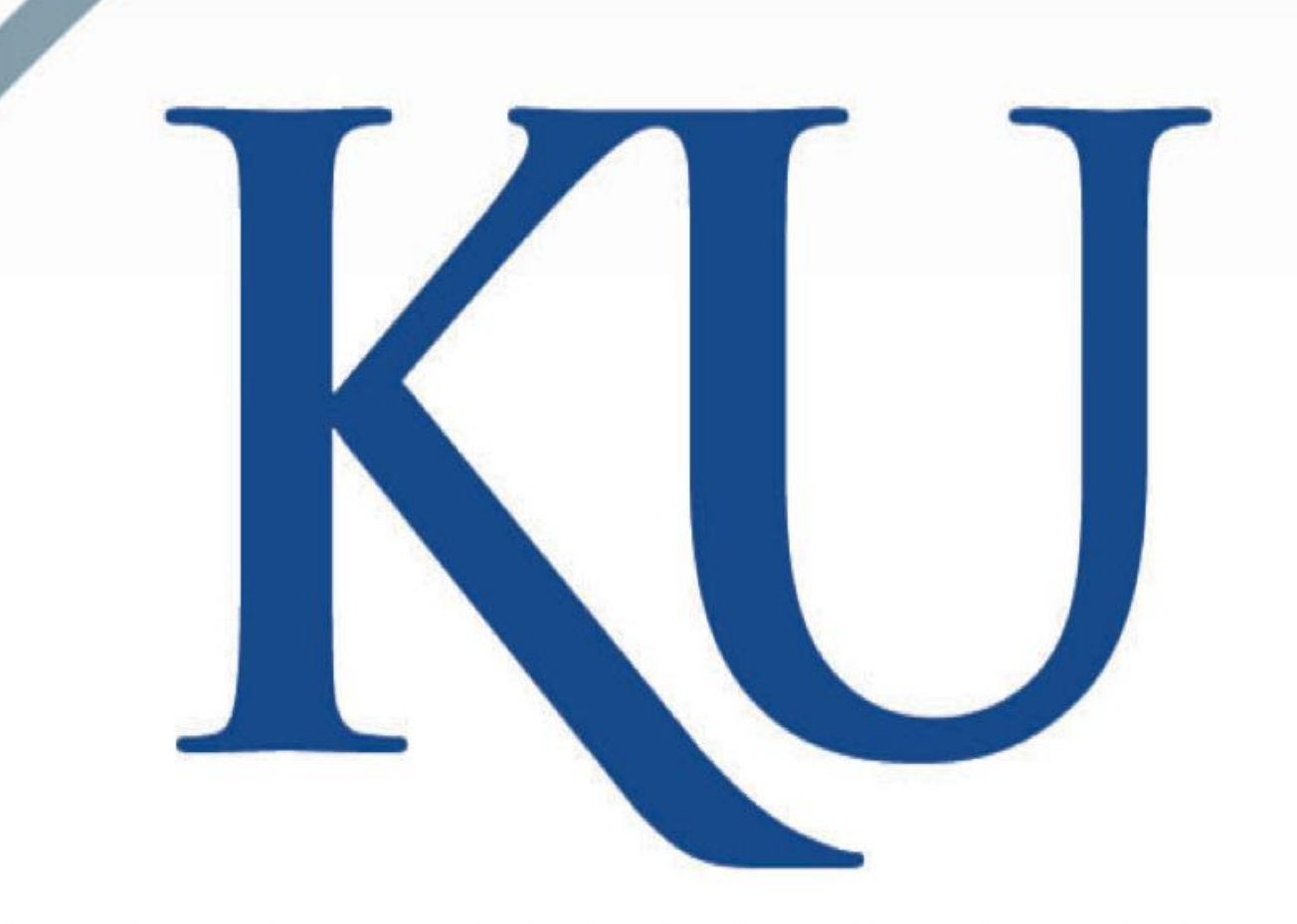

SCHOOL OF MEDICINE WICHTAA

The University of Kansas 